\title{
Total population study of factors affecting chronic bronchitis prevalence in the coal mining industry of New South Wales, Australia
}

\author{
J LEIGH, A N WILES, AND M GLICK \\ From the Joint Coal Board, Medical Division, Box 3842 GPO, Sydney, NSW 2001, Australia
}

ABSTRACT The period prevalence of simple chronic bronchitis (SCB) (mucus hypersecretion), defined as chronic cough and sputum production by the MRC respiratory symptom questionnaire administered by occupational physicians and of obstructive chronic bronchitis (OCB) (airflow obstruction) (defined as SCB plus $\mathrm{FEV}_{1}<80 \%$ predicted) have been measured over the period 30 June 1977-30 June 1980 in the entire work force aged between 21 and 60 of the coal industry of New South Wales, Australia (12357 men). Four dimensional contingency table analysis by a logistic transform method showed highly significant ( $p<0.001$ ) additive affects of age (exposure duration), site of work, smoking, and alcohol consumption on development of overall chronic bronchitis (SCB + OCB). Odds ratios were face work: surface work $=1 \cdot 78: 1$, smoker $:$ non-smoker $=4 \cdot 23: 1$, alcohol $>300 \mathrm{~g} / \mathrm{wk}$ : alcohol $<300 \mathrm{~g} / \mathrm{wk}=2 \cdot 13: 1$. There was no evidence for synergistic effects of these factors on the development of mucus hypersecretion. When OCB was analysed separately, the effect of site of work, although in the same direction, was not statistically significant and this was assumed to be due to a "healthy worker" effect or a "swamping" effect of smoking. Age, smoking, and alcohol effects were highly significant $(p<0.0001)$ and there was a sharp increase in prevalence of OCB in the age groups 41-50 and 51-60. Odds ratios were face work: surface work $=1 \cdot 11: 1$, smoker: non-smoker $=2 \cdot 66: 1$, alcohol $>300 \mathrm{~g} / \mathrm{wk}$ : alcohol $<300 \mathrm{~g} / \mathrm{wk}=2 \cdot 91: 1$. There was no evidence of synergistic effects. These results are consistent with a hypothesis of additive effects of smoking, alcohol, and coal mine dust and fumes on the development of chronic mucus hypersecretion leading to airflow obstruction or a hypothesis of similar additive effects on the development of two separate conditions-mucus hypersecretion with airflow obstruction and mucus hypersecretion without airflow obstruction.

The relative importance of occupational factors in the coal industry in the aetiology of simple chronic bronchitis (SCB) and obstructive chronic bronchitis (OCB) is still undecided. ${ }^{1}$ Studies in the United Kingdom show a progressive decrement of $\mathrm{FEV}_{1}$ with increasing exposure to dust, ${ }^{2-4}$ but only respirable dust $(1-5 \mu)$ was quantified, and it is thought that particles $>5 \mu$ are more likely to be important in causing bronchitis. ${ }^{5}$ In the United States a decreasing gradient of bronchitis prevalence between face, underground non-face, and surface workers has been shown, most obviously in non-smokers. ${ }^{5}$ Similar findings have been reported from West Germany. ${ }^{6}$ Further studies in the United States have shown a relation between indices of large airways obstruction and indices of dust exposure in non-smokers. ${ }^{7}$ Other studies, however, have shown less consistent effects. ${ }^{8-10}$

The coal mining industry in New South Wales has represented, except in the past 15 years, almost the whole bituminous coal industry in Australia. The New South Wales work force at present is about 20000 . Most mines are underground but in recent years open cut mining has increased and about $7 \%$ of the work force is currently employed in open cut mining. About $72 \%$ of the 8000 workers employed in Queensland mines are in open cut mines. Before 1970 the Queensland coal industry was negligible by comparison with that of New South Wales. Bituminous coal is mined to a very small extent in other parts of Australia. Most underground mining in New South 
Wales is bord and pillar but long wall systems are being increasingly used.

Thus the results in this paper, although based solely on New South Wales data, may be taken to reflect the evidence for coal mining effects on bronchitis in Australia as a whole over the period in which men have been exposed.

In 1948 the Joint Coal Board began a continuous occupational medical service. The examination includes a full clinical examination, doctor administered respiratory symptom questionnaire, detailed industrial history, smoking history, alcohol consumption history, spirometry, standard posterioanterior chest $x$ ray examination, and in some cases electrocardiography and exercise electrocardiography. The medical staff has been the same since 1977.

Medical bureaux in the mining districts keep lists of all employees. The men are scheduled for an examination every two to three years, by appointment in their working time. They get a shift off work for attending for a medical examination and non-attenders are vigorously followed up. The entire population at work in any given three year period will thus be examined with only a few isolated incidences of nonexamination $(<0 \cdot 1 \%)$.

Early sample surveys showed a gradient of decreasing prevalence of SCB and OCB between face, other underground, and surface workers. This gradient persisted when standardised for age and smoking and suggested a part coal dust aetiology for these conditions. ${ }^{11} 12$ It has now been possible to extend these studies to the entire work force.

The further opportunity was taken to examine the effects of alcohol on chronic bronchitis as recent experimental data has shown decreased mucociliary clearance with acute alcohol administration in $\operatorname{man}^{13}$ and alcohol consumption has also been shown to have relations with respiratory disease symptoms. ${ }^{14}$

\section{Methods}

The prevalence period was 30 June 1977 to 30 June 1980. All workers who worked throughout the period were examined. Workers who had joined the work force in the period and men under 21 or over 60 (retiring age) were excluded from the study. Workers who had retired or died in the period were included if they had been examined in the period. Men under 21 were excluded to be consistent with the study of Rogan et al 1973. ${ }^{2}$ These workers excluded men under 25 on the grounds that ventilatory capacity increases with age until a peak is reached between the ages of 21 and 25. They were considering $\mathrm{FEV}_{1}$ as the dependent variable in a multiple regression equation and presumably wished to avoid non-linear effects. We did not use this approach but compared actual FEV FE $_{1} \frac{\mathrm{a}}{\mathrm{O}}$ with a predicted value dependent on age. The predic- $\stackrel{\circ}{\circ}$ tion equations were based on data from subjects aged $\Rightarrow$

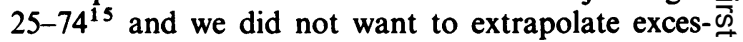
sively, while maintaining equal sized age groups. The 0 number of workers under 21 excluded was 891 .

Examinations were conducted by occupational $\frac{\bar{\sigma}}{\overline{0}}$ physicians and technicians whose techniques had $\frac{\mathbb{}}{\varnothing}$ been previously standardised. Data were recorded on standardised data entry forms, coded centrally, and ${ }_{3}^{\text {s }}$ entered into computer files via intelligent data entry? terminals. Details of the computer system have been $\overrightarrow{\vec{\omega}}$ previously published. ${ }^{16}$

\section{Data set}

(1) AGE(YEARS)

(2) SITE OF WORK

Underground (face).

Underground (non-face).

Surface (includes open cut workers).

Except in a few cases, present site of work was the $\frac{7}{0}$ same as site of work since entering the industry. This is so because of the nature of labour employment in $\vec{\bullet}$ the coal mining industry.

After a training period workers specialise in either face work, other underground support work, or sur-o face work and remain in this job for most of their working lives. A few older miners (aged 55-60) through disability or choice may move from face to $\frac{\mathrm{Q}}{\square}$ other underground or surface work.

Thus, in general, age may be taken as an index of $\overrightarrow{\overrightarrow{0}}$ years worked and hence exposure to industrial dust, 3 diesel, and shot firing fumes. Face work is known to have the highest concentration of dust and shot firing? fumes. Other underground work also entails exposure $\bar{O}$ to dust and fumes in lower concentration than at the $\frac{\mathbb{}}{3}$ face.

Surface workers are exposed to environmental con - . centrations similar to the general community in which mines are located. In New South Wales mines are located largely around two industrial cities, Newcastle을 (410250 population) and Wollongong (233650 popu- $>$ lation).

\section{(3) CATEGORYOF BRONCHITIS}

(1) Chronic cough and sputum production as evi- N denced by responses to questions on MRC respira- N tory symptom questionnaire (1966) administered by occupational physician.

The questions in the MRC questionnaire used $\frac{0}{\Phi}$ were:

Cough 1-Do you usually cough first thing in the 0 morning, or on getting up, in the winter?

Cough 3-Do you usually cough during the day (or at night) in the winter? 
Cough 5-Do you cough like this on most days (or nights) for as much as three months each year?

Phlegm 6-Do you usually bring up any phlegm from your chest first thing in the morning (or on getting up) in the winter?

Phlegm 8-Do you usually bring up any phlegm from your chest during the day (or at night) in the winter?

Phlegm 10-Do you bring up phlegm like this on most days (or nights) for as much as three months each year?

Bronchitis grade 1 required positive responses to: cough 1 (and/or) cough 3 and cough 5 plus phlegm 6 (and/or) phlegm 8 and phlegm 10. (Estimated sputum of $>2 \mathrm{cc} /$ day (total) required for positive response).

(2) (1) plus history of recurrent bronchial infection.

(3) (1) plus $60 \%<\mathrm{FEV}_{1}<80 \%$ predicted from equations of Ferris et al. ${ }^{15}$

(4) (1) plus $40 \%<\mathrm{FEV}_{1}<60 \%$ predicted.

(5) (1) plus $\mathrm{FEV}_{1}<40 \%$ predicted.

Categories 1 and 2 are SCB.

Category 3, 4, and 5 are OCB.

Catgories $1-5$ are mutually exclusive.

$\mathrm{FEV}_{1}$ was measured by Vitalograph spirometer as the better of two satisfactory efforts.

\section{SMOKING (FROM HISTORY)}

(1) Current smoker-g/wk tobacco (manufactured cigarette, handrolled, pipe and cigars included).

(2) Ex-smoker.

(3) Lifelong non-smoker.
Table 1 Prevalence of chronic bronchitis. (Percentages denote prevalence)

\begin{tabular}{lllll}
\hline Site & Non-B & $S C B$ & $O C B$ & Total \\
\hline Face & 4244 & 786 & $\begin{array}{l}165 \\
(15 \cdot 1 \%)\end{array}$ & 5195 \\
$\begin{array}{l}\text { Underground } \\
\text { non-face }\end{array}$ & 3152 & $\begin{array}{l}497 \\
(13 \cdot 1 \%)\end{array}$ & $\begin{array}{l}(4 \cdot 2 \%) \\
105\end{array}$ & 3808 \\
$\begin{array}{l}\text { Surface } \\
\text { (3.1\%) }\end{array}$ & 2905 & $\begin{array}{l}344 \\
(10 \cdot 3 \%)\end{array}$ & \begin{tabular}{l}
$(3.1 \% 4$ \\
\hline
\end{tabular} \\
\hline
\end{tabular}

\section{ALCOHOL CONSUMPTION(CALCULATED FROM}

HISTORY) G/WK ETHANOL

Complete data for all these variables were obtained from all examinees. Four dimensional contingency tables were produced with ICL package $X$ on an ICL 2904 computer. Cox's method of logistic transform analysis was used to analyse statistical significance of results. $^{17}$

\section{Results}

The total population studied comprised 12357 men aged between 21 and 60 . Of these, 338 had some degree of radiological pneumoconiosis (ILO 1980 categories $1 / 0: 196 ; 1 / 1: 125 ; 1 / 2: 8 ; 2 / 1: 6 ; 2 / 2: 3$ ) and these men were included in the study. Rogan et al have shown that pneumoconiosis has no effect on relations between chronic bronchitis and other variables under consideration, ${ }^{2}$ and this is confirmed by extensive postmortem studies from this institution ${ }^{1819}$ and by other workers. ${ }^{20}$

The overall prevalence of SCB was $1627 / 12357=$ $13 \cdot 2 \%$ and of OCB $429 / 12357=3 \cdot 5 \%$.

Table 2 Age distribution of chronic bronchitis. (Percentages denote prevalence)

\begin{tabular}{|c|c|c|c|c|}
\hline \multirow[t]{2}{*}{ Bronchitis grade } & \multicolumn{4}{|l|}{ Age (years) } \\
\hline & $21-30$ & $31-40$ & $41-50$ & $51-60$ \\
\hline $\left.\begin{array}{l}0 \\
1 \\
2\end{array}\right\} S C B$ & $\left.\begin{array}{r}2319 \\
155 \\
4\end{array}\right\}(6.4 \%)$ & $\left.\begin{array}{r}3120 \\
338 \\
2\end{array}\right\}(9 \cdot 7 \%)$ & $\left.\begin{array}{r}2842 \\
544 \\
3\end{array}\right\}(15 \cdot 6 \%)$ & $\left.\begin{array}{r}2020 \\
573 \\
8\end{array}\right\}(20 \cdot 3 \%)$ \\
\hline $\left.\begin{array}{l}3 \\
4 \\
5\end{array}\right\}$ OCB & $\left.\begin{array}{l}3 \\
0 \\
0\end{array}\right\}(0 \cdot 1 \%)$ & $\left.\begin{array}{r}32 \\
0 \\
0\end{array}\right\}(0 \cdot 9 \%)$ & $\left.\begin{array}{r}125 \\
3 \\
2\end{array}\right\}(3 \cdot 7 \%)$ & $\left.\begin{array}{r}256 \\
6 \\
2\end{array}\right\}(9 \cdot 0 \%)$ \\
\hline
\end{tabular}

Table 3 Age distribution of work force. (Percentages denote prevalence)

\begin{tabular}{llllll}
\hline Site & \multicolumn{2}{l}{ Age (years) } & & Total \\
\cline { 2 - 5 } & $21-30$ & $31-40$ & $41-50$ & $51-60$ & \\
\hline Face & $1136(21 \cdot 9 \%)$ & $1577(30 \cdot 4 \%)$ & $1514(29 \cdot 1 \%)$ & $968(18 \cdot 6 \%)$ & 5195 \\
Underground non-face & $831(21 \cdot 8 \%)$ & $979(25 \cdot 7 \%)$ & $1033(27 \cdot 1 \%)$ & $965(25 \cdot 4 \%)$ & 3808 \\
Surface & $514(15 \cdot 3 \%)$ & $936(27 \cdot 9 \%)$ & $972(29 \cdot 0 \%)$ & $932(27 \cdot 8 \%)$ & 3354 \\
\hline
\end{tabular}


Table 4 Overall effect of smoking. (Percentages denote prevalence)

\begin{tabular}{llll}
\hline & Smoker & Ex-smoker & $\begin{array}{l}\text { Lifelong } \\
\text { non-smoker }\end{array}$ \\
\hline Non-CB & 4627 & 2031 & 3610 \\
SCB + OCB & 1540 & 288 & 261 \\
& $(25 \cdot 0 \%)$ & $(12 \cdot 4 \%)$ & $(6 \cdot 7 \%)$ \\
\hline
\end{tabular}

For comparison:

$\mathrm{UK}^{2}$ SCB $29 \cdot 7 \%(\mathrm{n}=3581$ face workers) OCB $6.9 \%$

$\mathrm{US}^{7} \mathrm{SCB}+$ OCB $38.4 \%(\mathrm{n}=6014)$

West Germany ${ }^{6}$ SCB + OCB $=13 \%(n=$ 9935)

Table 1 shows overall prevalence of SCB and OCB by site of work. Prevalence of SCB is highest in face workers, next in underground non-face workers, and least in surface workers whereas prevalence of OCB is greatest in underground non-face workers and the same in face and surface workers. Analysis of the age distribution and smoking habit distribution of bronchitis, however, shows that an occupational effect must be sought with these two factors held constant. Age distribution by bronchitis grade is shown in table 2 and age by site of work in table 3. SCB and OCB prevalence increases with age and the underground non-face and surface workers have a higher proportion of older men.

\section{SMOKING}

Prevalence of bronchitis is strongly related to smoking (table 4). Prevalence of all bronchitis is $25 \%$ in smokers, $12.4 \%$ in ex-smokers, and $6.7 \%$ in lifelong non-smokers. Preliminary analysis showed a non- $\bar{z}$ significant effect of amount smoked in a current $\stackrel{D}{\varrho}$ smoker, probably because of uniform smoking patterns in coal workers, and smoking was thus classified into three categories: current smokers, ex-smokers, and lifelong non-smokers.

Table 5 shows the prevalence of SCB and OCB by age, site of work, smoking, and alcohol consumption. Generally, prevalence of SCB increases with age, smoking, alcohol consumption, and dust exposure. Prevalence of OCB trends are similar but not as clear. The increase with age is lagged behind that of SCB and workplace selection has probably caused some men with disability to move from face work, thus obscuring dust exposure effects.

The logistic transform:

with variance

$$
z=\log _{e}((R+1 / 2) /(n-R+1 / 2))
$$

$$
\mathrm{v}=\frac{(\mathrm{n}+1)(\mathrm{n}+2)}{\mathrm{n}(\mathrm{R}+1)(\mathrm{n}-\mathrm{R}+1)}
$$

where $\mathrm{R}=$ frequency was applied to $\mathrm{SCB}+\mathrm{OCB}$ and OCB alone. Tables 6 and 7 show the transformed data. We analysed mucus hypersecretion (SCB + OCB) and airflow obstruction (OCB) as separate entities.

Factorial analysis enabled all effects of interest to be tested for significance. Tables 8 and 9 show selected logistic factorial standardised contrasts. In these contrasts the three levels of smoking and work site were assumed to be equally spaced.

It may be seen for SCB + OCB (table 8) that all main linear effects are highly significant $(p<0.0001)$. The odds ratio for smoker:non-smoker is $4 \cdot 23: 1$,

\begin{tabular}{|c|c|c|c|c|c|c|c|}
\hline \multirow[t]{3}{*}{ Site } & \multirow{3}{*}{\multicolumn{2}{|c|}{$\begin{array}{l}\text { Alcohol } \\
\text { consumption } \\
\text { (g/week) }\end{array}$}} & \multicolumn{5}{|l|}{ Age (years) } \\
\hline & & & \multicolumn{3}{|l|}{$21-30$} & \multicolumn{2}{|l|}{$31-40$} \\
\hline & & & Non & $E x$ & $S$ & Non & $E x$ \\
\hline Face & $\begin{array}{l}<300 \\
>300\end{array}$ & $\begin{array}{l}\text { SCB } \\
\text { OCB } \\
n \\
\text { SCB } \\
\text { OCB } \\
n\end{array}$ & $\begin{array}{r}9(2.3 \%) \\
0(0.0 \%) \\
383 \\
3(12.5 \%) \\
0(0.0 \%) \\
24\end{array}$ & $\begin{array}{r}4(3.8 \%) \\
1(0.9 \%) \\
104 \\
2(11.8 \%) \\
0(0.0 \%) \\
17\end{array}$ & $\begin{array}{r}63(12 \cdot 2 \%) \\
0(0.0 \%) \\
515 \\
15(16 \cdot 1 \%) \\
2(2 \cdot 1 \%) \\
93\end{array}$ & 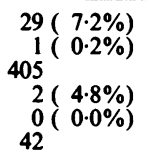 & $\begin{array}{r}20(9.0 \%) \\
2(0.9 \%) \\
223 \\
7(11.3 \%) \\
1(1.6 \%) \\
62\end{array}$ \\
\hline $\begin{array}{l}\text { Underground } \\
\text { non-face }\end{array}$ & $\begin{array}{l}<300 \\
>300\end{array}$ & $\begin{array}{l}\text { SCB } \\
\text { OCB } \\
\text { n } \\
\text { SCB } \\
\text { OCB } \\
\mathbf{n}\end{array}$ & $\begin{array}{r}9(2.6 \%) \\
0(0.0 \%) \\
352 \\
0(0.0 \%) \\
0(0.0 \%) \\
16\end{array}$ & $\begin{array}{l}3(3.6 \%) \\
0(0.0 \%) \\
83 \\
1(10.0 \%) \\
0(0.0 \%) \\
10\end{array}$ & $\begin{array}{r}28(12.1 \%) \\
0(0.0 \%) \\
232 \\
5(13.2 \%) \\
0(0.0 \%) \\
38\end{array}$ & $\begin{array}{r}10(3.5 \%) \\
0(0.0 \%) \\
287 \\
2(7.7 \%) \\
0(0.0 \%) \\
26\end{array}$ & $\begin{array}{r}6(4 \cdot 7 \%) \\
1(0.7 \%) \\
129 \\
4(16 \cdot 0 \%) \\
0(0.0 \%) \\
25\end{array}$ \\
\hline Surface & $\begin{array}{l}<300 \\
>300\end{array}$ & $\begin{array}{l}\text { SCB } \\
\text { OCB } \\
\text { n } \\
\text { SCB } \\
\text { OCB } \\
n\end{array}$ & $\begin{array}{r}3(1.5 \%) \\
0(0.0 \%) \\
203 \\
0(0.0 \%) \\
0(0.0 \%) \\
11\end{array}$ & $\begin{array}{l}1(2.1 \%) \\
0(0.0 \%) \\
47 \\
0(0.0 \%) \\
0(0.0 \%) \\
9\end{array}$ & 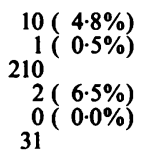 & $\begin{array}{r}8(2.9 \%) \\
1(0.4 \%) \\
277 \\
1(3.6 \%) \\
0(0.0 \%) \\
28\end{array}$ & $\begin{array}{r}3(2.3 \%) \\
1(0.8 \%) \\
130 \\
5(12.8 \%) \\
0(0.0 \%) \\
39\end{array}$ \\
\hline \multicolumn{8}{|c|}{ Non $=$ Lifelong non-smoker. Ex $=$ Current ex-smoker. $\mathbf{S}=$ Current smoker. $\mathbf{n}=$ Number in classification cell. } \\
\hline
\end{tabular}

Table 5 Prevalence (\%) of simple chronic bronchitis (SCB) and obstructive chronic bronchitis (OCB) 
able 6 Logistically transformed frequency data $(S C B+O C B)$

\begin{tabular}{|c|c|c|c|c|c|c|c|c|c|c|c|c|}
\hline \multirow[t]{3}{*}{ 'ite } & \multirow{3}{*}{$\begin{array}{l}\text { Alcohol } \\
\text { consump- } \\
\text { tion } \\
\text { (g/week) }\end{array}$} & \multicolumn{11}{|c|}{ Age (years) } \\
\hline & & \multicolumn{3}{|l|}{$21-30$} & \multicolumn{3}{|l|}{$31-40$} & \multicolumn{3}{|l|}{$41-50$} & \multicolumn{2}{|l|}{$51-60$} \\
\hline & & Non & $E x$ & Smoker & Non & $E x$ & Smoker & Non & $E x$ & Smoker & Non & $E x$ \\
\hline $\begin{array}{l}\text { Jnderground } \\
\text { non-face } \\
\text { urface }\end{array}$ & $\begin{array}{cc} & \mathrm{Z} \\
<300 & \mathrm{v} \\
& \mathrm{Z} \\
>300 & \mathrm{v} \\
& \mathrm{Z} \\
<300 & \mathrm{v} \\
& \mathrm{Z} \\
>300 & \mathrm{v} \\
& \mathrm{Z} \\
<300 & \mathrm{v} \\
& \mathrm{Z} \\
>300 & \mathrm{v}\end{array}$ & $\begin{array}{c}-3 \cdot 674 \\
0 \cdot 1029 \\
-1 \cdot 815 \\
0 \cdot 3078 \\
-3 \cdot 588 \\
0 \cdot 1032 \\
-3 \cdot 497 \\
1 \cdot 125 \\
-4 \cdot 048 \\
0 \cdot 2562 \\
-3 \cdot 135 \\
1 \cdot 182\end{array}$ & $\begin{array}{c}-2.895 \\
0.1784 \\
-1.825 \\
0.4191 \\
-3.135 \\
0.2665 \\
-1.846 \\
0.660 \\
-3.434 \\
0.5324 \\
-2.944 \\
1.222\end{array}$ & $\begin{array}{c}-1.964 \\
0.0179 \\
-1.475 \\
0.0693 \\
-2.369 \\
0.0266 \\
-1.807 \\
0.2012 \\
-2.853 \\
0.0888 \\
-2.468 \\
0.3785\end{array}$ & $\begin{array}{c}-2.510 \\
0.0350 \\
-2 \cdot 785 \\
0.3662 \\
-3.274 \\
0.0948 \\
-2.282 \\
0.3877 \\
-3.342 \\
0.1040 \\
-2.909 \\
0.555\end{array}$ & $\begin{array}{c}-2 \cdot 192 \\
0.0486 \\
-1.858 \\
0.1293 \\
-2.793 \\
0.1342 \\
-1.564 \\
0.2553 \\
-3.336 \\
0.2095 \\
-1.836 \\
0.2002\end{array}$ & $\begin{array}{c}-1.775 \\
0.0119 \\
-1.038 \\
0.0296 \\
-1.979 \\
0.0224 \\
-1.045 \\
0.0527 \\
-2.367 \\
0.0333 \\
-1.212 \\
0.0659\end{array}$ & $\begin{array}{c}-2.334 \\
0.0333 \\
-1.236 \\
0.0943 \\
-2.735 \\
0.0714 \\
-1.726 \\
0.1739 \\
-3.116 \\
0.0875 \\
-3.296 \\
0.5372\end{array}$ & $\begin{array}{c}-2.047 \\
0.0437 \\
-1.699 \\
0.1349 \\
-2.636 \\
0.0900 \\
-1.155 \\
0.1127 \\
-2.482 \\
0.0780 \\
-1.457 \\
0.1413\end{array}$ & $\begin{array}{c}-0.959 \\
0.0085 \\
-0.261 \\
0.0176 \\
-0.981 \\
0.0130 \\
-0.321 \\
0.0271 \\
-1.742 \\
0.0251 \\
-0.523 \\
0.0321\end{array}$ & $\begin{array}{c}-1.684 \\
0.0351 \\
-1.582 \\
0.1556 \\
-1.946 \\
0.0426 \\
-1.052 \\
0.0921 \\
-2.371 \\
0.0524 \\
-1.669 \\
0.1757\end{array}$ & $\begin{array}{c}-1.402 \\
0.032 \\
-0.909 \\
0.1030 \\
-1.421 \\
0.0313 \\
-0.676 \\
0.1036 \\
-1.539 \\
0.0371 \\
-1.144 \\
0.0899\end{array}$ \\
\hline \multicolumn{13}{|c|}{$\begin{array}{l}=\log _{f}((\mathrm{R}+1 / 2) /(\mathrm{n}-\mathrm{R}+1 / 2)) \quad \mathrm{v}=(\mathrm{n}+1)(\mathrm{n}+2) /(\mathrm{n}(\mathrm{R}+1)(\mathrm{n}-\mathrm{R}+1)) \\
\text { Vhere } \mathrm{R}=\text { number with SCB }+ \text { number with } \mathrm{OCB}, \mathrm{n}=\text { number in classification cell. } \\
\text { Table } 7 \text { Logistically transformed frequency data }(O C B)\end{array}$} \\
\hline \multirow[t]{3}{*}{ Site } & \multirow{3}{*}{$\begin{array}{l}\text { Alcohol } \\
\text { consump- } \\
\text { tion } \\
\text { (g/week) }\end{array}$} & \multicolumn{11}{|c|}{ Age (years) } \\
\hline & & \multicolumn{3}{|l|}{$21-30$} & \multicolumn{3}{|l|}{$31-40$} & \multicolumn{3}{|l|}{$41-50$} & \multicolumn{2}{|l|}{$51-60$} \\
\hline & & Non & $E x$ & Smoker & Non & $E x$ & Smoker & Non & $E x$ & Smoker & Non & $E x$ \\
\hline $\begin{array}{l}\text { Jnderground } \\
\text { non-face } \\
\text { Surface }\end{array}$ & $\begin{array}{cc} & \mathrm{z} \\
<300 & \mathrm{v} \\
& \mathrm{z} \\
>300 & \mathrm{v} \\
& \mathrm{z} \\
<300 & \mathrm{v} \\
& \mathrm{z} \\
>300 & \mathrm{v} \\
& \mathrm{z} \\
<300 & \mathrm{v} \\
& \mathrm{z} \\
>300 & \mathrm{v}\end{array}$ & $\begin{array}{c}-6.642 \\
1.0052 \\
-3.892 \\
1.0833 \\
-8.860 \\
1.0057 \\
-3.497 \\
1.1250 \\
-6.009 \\
1.0098 \\
-3.135 \\
1.1818\end{array}$ & $\begin{array}{c}-4.234 \\
0.5145 \\
-3.555 \\
1.1176 \\
-5.118 \\
1.0241 \\
-3.044 \\
1.2000 \\
-4.554 \\
1.0425 \\
-2.944 \\
1.2222\end{array}$ & $\begin{array}{c}-6.938 \\
1.0038 \\
-3.600 \\
0.3479 \\
-6.142 \\
1.0086 \\
-4.344 \\
1.0526 \\
-4.939 \\
0.5071 \\
-4 \cdot 143 \\
1.0645\end{array}$ & $\begin{array}{c}-5.597 \\
0.5037 \\
-4.443 \\
1.0476 \\
-6.354 \\
1.0069 \\
-3.970 \\
1.0769 \\
-5.217 \\
0.5054 \\
-4.043 \\
1.0714\end{array}$ & $\begin{array}{c}-4.484 \\
0.3394 \\
-3.714 \\
0.5244 \\
-4.450 \\
0.5117 \\
-3.932 \\
1.0800 \\
-4.458 \\
0.5116 \\
-4.369 \\
1.0513\end{array}$ & $\begin{array}{c}-3.748 \\
0.0641 \\
-3.422 \\
0.1736 \\
-4.313 \\
0.1695 \\
-3.643 \\
0.3473 \\
-5.015 \\
0.3368 \\
-5.130 \\
1.0238\end{array}$ & $\begin{array}{c}-4.392 \\
0.2033 \\
-3.135 \\
0.3565 \\
-4.545 \\
0.3390 \\
-4.443 \\
1.0476 \\
-4.336 \\
0.2547 \\
-4.418 \\
1.0488\end{array}$ & $\begin{array}{c}-3.506 \\
0.1481 \\
-3.574 \\
0.5281 \\
-4.733 \\
0.5088 \\
-3.433 \\
0.5324 \\
-3.892 \\
0.2573 \\
-4.489 \\
1.0455\end{array}$ & $\begin{array}{c}-2.766 \\
0.0304 \\
-2.384 \\
0.0549 \\
-2.652 \\
0.0413 \\
-2 \cdot 168 \\
0.0703 \\
-3.575 \\
0.1147 \\
-2.457 \\
0.0998\end{array}$ & $\begin{array}{c}-3.463 \\
0.1484 \\
-2.809 \\
0.3654 \\
-3.454 \\
0.1484 \\
-2.438 \\
0.2232 \\
-3.434 \\
0.1298 \\
-2.093 \\
0.2327\end{array}$ & $\begin{array}{c}-2.972 \\
0.1059 \\
-1.830 \\
0.1672 \\
-2.909 \\
0.0966 \\
-2.424 \\
0.2815 \\
-2.536 \\
0.0777 \\
-2.512 \\
0.2216\end{array}$ \\
\hline & \multicolumn{6}{|c|}{$41-50$} & \multicolumn{4}{|l|}{$51-60$} & & \\
\hline$S$ & \multicolumn{2}{|l|}{ Non } & \multicolumn{2}{|l|}{$E x$} & \multicolumn{2}{|l|}{$S$} & \multicolumn{2}{|l|}{ Non } & \multicolumn{2}{|l|}{$E x$} & \multicolumn{2}{|l|}{$S$} \\
\hline $\begin{array}{l}82(12 \cdot 2 \%) \\
15(2 \cdot 2 \%) \\
572 \\
40(23 \cdot 1 \%) \\
5(2.9 \%) \\
173(\end{array}$ & \multicolumn{2}{|c|}{$\begin{array}{r}28(7.6 \%) \\
4(1.1 \%) \\
367 \\
11(18.6 \%) \\
2(3.4 \%) \\
59\end{array}$} & \multicolumn{2}{|c|}{ 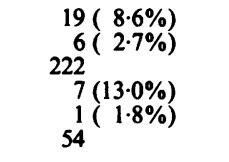 } & \multicolumn{2}{|c|}{$\begin{array}{r}127(21 \cdot 8 \%) \\
34(5 \cdot 8 \%) \\
582 \\
81(35 \cdot 2 \%) \\
19(8.3 \%) \\
230\end{array}$} & \multicolumn{2}{|c|}{$\begin{array}{r}27(12 \cdot 7 \%) \\
6(2 \cdot 8 \%) \\
213 \\
5(11 \cdot 6 \%) \\
2(4 \cdot 6 \%) \\
43\end{array}$} & \multicolumn{2}{|c|}{$\begin{array}{r}29(14.9 \%) \\
9(4.6 \%) \\
194 \\
7(15.2 \%) \\
6(13.0 \%) \\
46\end{array}$} & \multicolumn{2}{|c|}{$\begin{array}{r}108(31 \cdot 7 \%) \\
39(11 \cdot 4 \%) \\
341 \\
55(41 \cdot 9 \%) \\
29(22 \cdot 1 \%) \\
131\end{array}$} \\
\hline $\begin{array}{r}45(10 \cdot 8 \%) \\
5(1 \cdot 2 \%) \\
415 \\
23(23 \cdot 7 \%) \\
2(2 \cdot 1 \%) \\
97\end{array}$ & \multicolumn{2}{|c|}{$\begin{array}{r}12(5.1 \%) \\
2(0.8 \%) \\
237 \\
6(14.3 \%) \\
0(0.0 \%) \\
42\end{array}$} & \multicolumn{2}{|c|}{$\begin{array}{r}10(5.8 \%) \\
1(0.6 \%) \\
171 \\
10(21 \cdot 3 \%) \\
1(2 \cdot 1 \%) \\
47\end{array}$} & \multicolumn{2}{|c|}{$\begin{array}{l}80(20 \cdot 7 \%) \\
25(6.5 \%) \\
386 \\
48(32 \cdot 0 \%) \\
15(10.0 \%) \\
150\end{array}$} & \multicolumn{2}{|c|}{$\begin{array}{r}20(9.5 \%) \\
6(2.8 \%) \\
211 \\
10(18.2 \%) \\
4(7.3 \%) \\
55\end{array}$} & \multicolumn{2}{|c|}{$\begin{array}{r}29(14 \cdot 3 \%) \\
10(4.9 \%) \\
202 \\
11(26 \cdot 2 \%) \\
3(7 \cdot 1 \%) \\
42\end{array}$} & $\begin{array}{c}88(27 \cdot 5 \\
44(13 \cdot 7 \\
320 \\
59(43 \cdot 7 \\
24(17 \cdot 8 \\
135\end{array}$ & $\begin{array}{l}\% \%) \\
\% \%) \\
\% \text { ) }\end{array}$ \\
\hline $\begin{array}{l}30(7.9 \%) \\
2(0.5 \%) \\
378 \\
19(22.6 \%) \\
0(0.0 \%) \\
84\end{array}$ & $\begin{array}{r}8 \\
3( \\
270 \\
1 \\
0 \\
41\end{array}$ & $\begin{array}{l}(3.0 \%) \\
(1.1 \%) \\
(2.4 \%) \\
0.0 \%)\end{array}$ & $\begin{array}{r}10 \\
3( \\
174 \\
8(1 \\
0( \\
44\end{array}$ & $\begin{array}{l}5 \cdot 7 \%) \\
1 \cdot 7 \%) \\
\\
8 \cdot 2 \%) \\
0 \cdot 0 \%)\end{array}$ & $\begin{array}{l}38(1) \\
8( \\
311 \\
39(2 \\
10( \\
132\end{array}$ & $\begin{array}{l}2 \cdot 2 \%) \\
2 \cdot 6 \%) \\
9 \cdot 5 \%) \\
7 \cdot 6 \%)\end{array}$ & $\begin{array}{r}13(5) \\
7(5) \\
239 \\
2(5) \\
4(10 . \\
40\end{array}$ & $\begin{array}{l}.4 \%) \\
.0 \%) \\
.0 \%)\end{array}$ & $\begin{array}{l}19(10 . \\
13(7 . \\
183 \\
10(16 . \\
4(6 . \\
59\end{array}$ & $\begin{array}{l}4 \%) \\
1 \%) \\
9 \%) \\
8 \%)\end{array}$ & $\begin{array}{r}72(24 \cdot 6 \\
39(13 \cdot 3 \\
293 \\
40(33 \cdot 8 \\
25(21 \cdot 2 \\
118\end{array}$ & \\
\hline
\end{tabular}


Table 8 Selected logistic factorial standardised contrasts $(S C B+O C B) .(u$ is a standard normal deviate)

\begin{tabular}{|c|c|c|c|}
\hline & $u$ & $p$ & Odds ratios \\
\hline Age (linear effect) & $11 \cdot 72$ & $<0.0001$ & $\begin{array}{l}51-60: 41-50=2 \cdot 11: 1 \\
41-50: 31-40=1 \cdot 69: 1 \\
31-40: 21-30=1 \cdot 61: 1\end{array}$ \\
\hline Age (quadratic effect) & $1 \cdot 34$ & NS & \\
\hline Site (linear effect) & $4 \cdot 75$ & $<0.0001$ & $\begin{array}{l}\text { Face }: \text { surface }=1 \cdot 78: 1 \\
\text { Face }: \text { underground }=1 \cdot 19: 1 \\
\text { Underground }: \text { surface }=1 \cdot 50: 1\end{array}$ \\
\hline Site (quadratic effect) & $-1 \cdot 14$ & NS & \\
\hline Smoking (linear effect) & $12 \cdot 69$ & $<0.0001$ & $\begin{array}{l}\text { Smoker }: \text { non-smoker }=4 \cdot 23: 1 \\
\text { Smoker }: \text { ex-smoker }=2 \cdot 42: 1 \\
\text { Ex-smoker }: \text { non-smoker }=1 \cdot 75: 1\end{array}$ \\
\hline Smoking (quadratic effect) & -1.48 & NS & \\
\hline Alcohol main effect & $7 \cdot 65$ & $<0.0001$ & Alcohol $>300 \mathrm{~g} / \mathrm{wk}:$ alcohol $<300 \mathrm{~g} / \mathrm{wk}=2 \cdot 13: 1$ \\
\hline Site (linear) $\times$ age (linear) & $1 \cdot 27$ & NS & \\
\hline Site (linear) $\times$ age (quadratic) & 0.03 & NS & \\
\hline Site (linear) $\times$ smoking (linear) & -0.89 & NS & \\
\hline Site (linear) $\times$ alcohol & -0.31 & NS & \\
\hline Smoking (linear) $\times$ age (linear) & 1.93 & NS & \\
\hline Alcohol $x$ age (linear) & -0.45 & NS & \\
\hline Smoking (linear) $\times$ alcohol & 0.58 & NS & \\
\hline Age $($ linear $) \times$ smoking $($ linear $) \times$ alcohol & 0.79 & NS & \\
\hline Site (linear) $\times$ smoking (linear $) \times$ alcohol & $-0 \cdot 71$ & NS & \\
\hline Age (linear) $\times$ site (linear) $\times$ alcohol & $-0 \cdot 40$ & NS & \\
\hline Age (linear) $\times$ site (linear) $\times$ smoking (linear) & -0.51 & NS & \\
\hline Age (linear) $\times$ site (linear) $\times$ smoking (linear) $\times$ alcohol & $0 \cdot 52$ & NS & \\
\hline
\end{tabular}

compared with $1 \cdot 78: 1$ for face : surface and $2 \cdot 13: 1$ for heavy drinker : light drinker. No interactions or nonlinear effects tested were significant. The data thus are consistent with a hypothesis of additive effects of age, smoking, dust, and alcohol on the development of chronic mucus hypersecretion.

When OCB was considered separately (table 9), the linear age effect was highly signiticant $(\mathrm{p}<0.0001)$ and in addition there was a positive quadratic effect $(p$ $<0.01)$ reflecting the sharper increase in OCB in the higher age group. The smoking effect was highly significant but the odds ratio less than that for SCB + OCB, possibly reflecting discontinuation of smoking habit by those affected. The alcohol effect was more

Table 9 Selected logistic factorial standardised contrasts (OCB). ( $\mathrm{u}$ is a standard normal deviate)

\begin{tabular}{|c|c|c|c|}
\hline & $u$ & $p$ & Odds ratios \\
\hline Age (linear) & $10 \cdot 23$ & $<0.0001$ & $\begin{array}{l}51-60: 41-50=3 \cdot 44: 1 \\
41-50: 31-40=2 \cdot 35: 1 \\
31-40: 21-30=1 \cdot 34: 1\end{array}$ \\
\hline Age (quadratic) & $2 \cdot 75$ & $<0.01$ & \\
\hline Age (cubic) & -0.24 & NS & \\
\hline Site (linear) & 0.51 & NS & \\
\hline Site (quadratic) & $1 \cdot 05$ & NS & \\
\hline Smoking (linear) & $4 \cdot 81$ & $<0.0001$ & $\begin{array}{l}\text { Smoker }: \text { non-smoker }=2 \cdot 66: 1 \\
\text { Smoker }: \text { ex-smoker }=1 \cdot 31: 1 \\
\text { Ex-smoker }: \text { non-smoker }=2 \cdot 02: 1\end{array}$ \\
\hline Smoking (quadratic) & $-1 \cdot 17$ & NS & \\
\hline Alcohol main effect & $6 \cdot 24$ & $<0.0001$ & Alcohol $>300 \mathrm{~g} / \mathrm{wk}:$ alcohol $<300 \mathrm{~g} / \mathrm{wk}=2.91: 1$ \\
\hline Site (linear) $\times$ age (linear) & 0.73 & NS & \\
\hline Site (linear) $\times$ age (quadratic) & $2 \cdot 04$ & $<0.05$ & \\
\hline Site (linear) $\times$ smoking (linear) & 1.02 & NS & \\
\hline Site (linear) $\times$ alcohol & 0.89 & NS & \\
\hline Smoking (linear) $\times$ age (linear) & $2 \cdot 03$ & $<0.05$ & \\
\hline Smoking (linear) $\times$ age (quadratic) & -0.78 & NS & \\
\hline Alcohol $x$ age (linear) & $-3 \cdot 12$ & $<0.002$ & \\
\hline Alcohol $\times$ age (quadratic) & $2 \cdot 50$ & $<0.02$ & \\
\hline Smoking (linear) $\times$ alcohol & -1.95 & $\sim 0.05$ & \\
\hline Smoking (linear) $\times$ site (linear) $\times$ alcohol & 0.51 & NS & \\
\hline Age (linear) $\times$ smoking (linear) $\times$ alcohol & 1.40 & NS & \\
\hline Age (quadratic) $\times$ smoking (linear) $\times$ alcohol & -0.67 & NS & \\
\hline Age (linear) $\times$ site (linear) $\times$ alcohol & $0 \cdot 22$ & NS & \\
\hline Age (quadratic) $\times$ site (linear) $\times$ alcohol & 0.02 & NS & \\
\hline Age (linear) $\times$ site (linear) $\times$ smoking (linear) & $-0 \cdot 23$ & NS & \\
\hline Age (quadratic) $\times$ site (linear) $\times$ smoking (linear) & -0.73 & NS & \\
\hline $\begin{array}{l}\text { Age (linear) } \times \text { smoking (linear) } \times \text { site (linear) } \times \text { alcohol } \\
\text { Age (quadratic) } \times \text { smoking (linear) } \times \text { site }(\text { linear })\end{array}$ & -0.94 & NS & \\
\hline$\times$ alcohol & $1 \cdot 34$ & NS & \\
\hline
\end{tabular}


pronounced than that for SCB + OCB. The borderline interactions between age and smoking and age and site may be ignored as some data cells contain small numbers or are empty and this affects the accuracy of the approximations involved in the analysis. The interaction between alcohol and age (linear and quadratic) may be seen from inspection of table 5 to result from no fixed pattern of association and we regard it as spurious. There is a borderline negative interaction between smoking and alcohol and again this probably is spurious. (Some ex-smokers appear to have less risk of obstructive disease if they drink more.)

The main difference between the separate analysis of OCB and that of SCB + OCB is the lack of significant site effect. Inspection of table 5 shows that in those aged 51-60 OCB prevalence is fairly uniform in different sites, being slightly greater in surface workers. This is a healthy worker effect common to studies of this type. Following the suggestion of Peto et al, ${ }^{21}$ we looked at the age groups $31-40,41-50$, and then the gradient face-underground non-face-surface of OCB prevalence is more apparent (table 5)-that is, the healthy worker effect has not yet operated but $\mathrm{FEV}_{1}$ loss has occured. Even in these restricted age groups, however, the site gradient was not statistically significant $(p>0.05)$.

\section{Discussion}

This study provides evidence for a dust exposure related aetiology for SCB and, to a lesser extent, for OCB in coal miners. It is the first reported total population study of these conditions in an industry population and the population studied is larger than the largest sample survey so far available. It is also the first major report on these conditions from the Australian coal industry. The results are consistent with the findings of Rogan et al and Rae et al in the British coal industry ${ }^{2}$ and those of Kibelstis et al and Hankinson et al in the United States. ${ }^{57}$ The overall prevalence of SCB and OCB is lower in Australia and this may be related to better dust conditions in the coal mines and less atmospheric pollution in coal mining areas. The New South Wales coal industry is only now introducing personal gravimetric dust sampling and has previously maintained a particle count dust monitoring system. Although it may have been possible to obtain better estimates of individual exposure from industrial history and average mine dust counts, this would have been a difficult exercise and we thought that any increased accuracy obtained would not justify the effort. The study would still be subject to the criticism that only respirable particles are counted and the inevitable confounding of exposure with age. With an estimated dust exposure for each individual it might have been possible to carry out a more quantitative study using either clinical SCB grade or actual $\mathrm{FEV}_{1}$ as the dependent variable and dust exposure, smoking, alcohol, age, and height as independent variables.

This would have implied an accuracy of history taking greater than justified, however, and interpretation would have been made difficult by correlations between smoking, drinking, age, and exposure. We thought it better to use "bronchitis" diagnosis by occupational physicians rather than $\mathrm{FEV}_{1}$ for the dependent variable as minor degrees of FEV $_{1}$ loss may have been due to other lung conditions. Although a cross sectional study cannot provide data on the natural history of a disease, the results of this study are consistent with the hypothesis that OCB is a sequel to SCB. When OCB is analysed separately (table 5 ) the prevalence in all smoking groups is lower than that of SCB, and increases with age and smoking. The increase with age is lagged behind SCB. The gradient with site of work is less clear than with SCB but the difference between face

Table 10 Changes in prevalence* of $S C B, O C B$, and pneumoconiosis with time

\begin{tabular}{|c|c|c|c|c|c|c|c|}
\hline & 1948 & 1954 & $1957-60$ & $1963-5$ & $1971-4$ & $1974-7$ & $1977-80$ \\
\hline $\begin{array}{l}\text { All chronic bronchitis } \\
(\mathrm{SCB}+\mathrm{OCB})(\%)\end{array}$ & $33 \cdot 0_{+}^{+}$ & $38 \cdot 0 \pm$ & $25.0 \|$ & $21 \cdot 0$ & $17 \cdot 6$ & $13 \cdot 6$ & $12 \cdot 8$ \\
\hline $\begin{array}{l}\text { Obstructive chronic } \\
\text { bronchitis (OCB) }(\%)\end{array}$ & $9 \cdot 0 \ddagger$ & $14 \cdot 0_{+}^{+}$ & $10 \cdot 0 \|$ & 6.0 & $4 \cdot 6$ & $3 \cdot 3$ & $2 \cdot 8$ \\
\hline $\begin{array}{l}\text { Smokers (\%) } \\
\text { Pneumoconiosis (\%) } \\
\quad \text { ILO } \geqslant 1 / 0\end{array}$ & $\begin{array}{l}\text { NA } \\
16.0\end{array}$ & $\begin{array}{c}62 \\
8 \cdot 7\end{array}$ & $\begin{array}{r}65 \| \\
3.6\end{array}$ & $\begin{array}{l}62 \\
3.0\end{array}$ & $\begin{array}{l}72 \\
3 \cdot 0\end{array}$ & $\begin{array}{l}67 \\
2 \cdot 9\end{array}$ & $\begin{array}{c}63 \\
2 \cdot 0\end{array}$ \\
\hline $\begin{array}{l}\% \text { NSW total work force } \\
\text { examined } \dagger\end{array}$ & $10 \%$ & $10 \% \S$ & $85 \%$ & $60 \%$ & $100 \%$ & $100 \%$ & $100 \%$ \\
\hline
\end{tabular}

* For consistency with earlier surveys, prevalence figures relate to populations with new entrants included.

tAverage total work force 17000 .

†OCB assessed by clinical criteria.

\$Southern district (Wollongong) only.

$\| 10 \%$ sample.

NA $=$ Not available. 
and surface work is apparent in each age group except the oldest. Prevalence differences in this group have been obscured by smoking and work force movements which would be in a direction such as to underestimate work site effects.

Recently published longitudinal studies on smaller groups than that in the present study now appear to show that chronic mucus hypersecretion and airflow obstruction should be regarded as two distinct conditions with a common aetiology (mainly smoking), ${ }^{21-23}$ and our data are also consistent with this hypothesis.

The prevalence of SCB and OCB in non-smoking surface workers may be taken to estimate the community prevalence without smoking or dust effects. For comparison with other studies, over all age groups, this was $4.6 \%$. Jacobsen found that the prevalence ratio between smoking low dust exposed workers and non-smoking low dust exposed workers was 34\%/9\% (all ages). ${ }^{24}$ The comparable figures in this study are $27 \cdot 4 \% / 4 \cdot 8 \%$ for surface workers (table 5 ) giving a

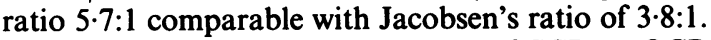
Among all smokers, the prevalence of SCB + OCB was $25.0 \%$ compared with $6.7 \%$ for lifelong nonsmokers (3.7:1, cf Jacobsen $44 \%$ compared with $18 \%$, 2:4:1).

The low prevalence of cough and sputum plus infection (chronic bronchitis grade 2 ) is probably related to the generally affluent living conditions that miners enjoy outside work and the low atmospheric pollution in New South Wales mining areas. Miners with grades 4 and 5 chronic bronchitis would be disabled from work in general and the low prevalence in the working population is due to retirement.

The alcohol effect has not been previously reported in a coal mining population but similar findings have been reported by Lebowitz. ${ }^{14} \mathrm{He}$ found the "bronchitis" prevalence ratio between "heavy" ( $>6.25$ US oz, $(184 \mathrm{~g}))$ and light or moderate drinking in lifelong non-smokers was $21 \cdot 5 \% / 15 \cdot 2 \%$ (aged adjusted) compared with $19.6 \% / 11.9 \%$ in the $51-60$ age group and $14 \cdot 1 \% / 6 \cdot 8 \%$ in the $41-50$ age group in the present study. We thought that to define heavy drinking as $>184 \mathrm{~g} / \mathrm{wk}$ would be too low a criterion for an all male heavy industry population and chose higher limits accordingly.

Sample and total population surveys of bronchitis prevalence in New South Wales coal miners have been carried out since $1948 .^{111225}$ Table 10 shows that SCB and OCB prevalence has been falling continuously whereas smoking habits have not changed significantly. Pneumoconiosis prevalence has also been falling, reflecting better dust control (as well as work force redeployment). A sharp fall in prevalence around 1960 coincided with the elimination of shot firing in coal mines and its associated nitrogen oxides.
This time trend is also consistent with an industrial aetiology for bronchitis.

There is significant large airways obstruction in coal workers. Hankinson et al found better relations $\stackrel{\vec{P}}{\stackrel{P}{\mathscr{S}}}$ between indices of large rather than small airways $\bar{c}$ obstruction and dust exposure. ${ }^{7}$ Postmortem measurements from this institution show that gland/wall $\frac{\bar{\rho}}{\vec{\sigma}}$ ratio and wall internal to cartilage/lumen ratio in $\underset{\mathbb{Q}}{\mathbb{2}}$ large bronchi correlated well with symptoms and signs of OCB. ${ }^{26}$ The prevalence of gland/wall ratio $>0.4$ in the postmortem sample is $46 \%$ and $?$ gland/wall ratio is not correlated with postmortem $\overrightarrow{\vec{\omega}}$ pneumoconiosis. ${ }^{1819}$

In other necropsy studies significant relations between coal mine dust exposure, smoking, and bron- 3 chial gland enlargement were found by Douglas $\vec{\omega}$ et al. ${ }^{20}$

We are currently carrying out retroprospective studies similar to those of Love and Miller ${ }^{4}$ relating $\omega$ quantified aetiological factors in individuals to the $\bigcirc$ progression of simple and obstructive chronic bron- $\rightarrow$ chitis (mucus hypersecretion and airflow obstruc- $\frac{D}{0}$ tion). We hope, however, that in future reduced $=$ smoking habit and maintained dust control will keep $\overrightarrow{0}$ industrial bronchitis in the New South Wales coal $\%$ industry to a minimum. The Queensland coal industry has started a medical surveillance scheme identical to that of New South Wales and data will soon be available for comparison. It should be noted, however, that with increased use of long wall mining techniques and possible increased dust production, constant epidemiological monitoring of industrial lung disease will be required.

We thank all the staff of the medical and EDP? divisions of the Joint Coal Board for their help in accurate collection and processing of data, and a ref- $\frac{}{3}$ eree of an earlier version of this paper for helpful advice on statistical analysis.

\section{References}

${ }^{1}$ Morgan WKC. Industrial bronchitis. $\mathrm{Br} J$ Ind Med 1978; 을 35:285-91.

2 Rogan JM, Attfield MD, Jacobsen M, Rae S, Walker DD, Walton $\mathrm{N}$ WH. Role of dust in the working environment in development of chronic bronchitis in British coal miners. Br J Ind Med 1973;30:217-26.

${ }^{3}$ Rae S, Walker DD, Attfield MD. Chronic bronchitis and dust $\omega$ exposure in British coal miners. In: Walton WH, ed. Inhaled particles III. Old Woking, Surrey: Unwin, 1971:883-94.

${ }^{4}$ Love RG, Miller BG. Longitudinal study of lung function in coal miners. Thorax 1982;37:193-7.

${ }^{5}$ Kibelstis JA, Morgan EJ, Reger R, Lapp NL, Seaton A, Morgan WKC. Prevalence of bronchitis and airway obstruction in American bituminous coal miners. Am Rev Respir Dis 1973;108:886-93

${ }^{6}$ Smidt U. Dust and non-specific respiratory disorders in foundry 
workers and coal miners in the Rhine-Ruhr area. Revue de L'Institut d'Hygiène des Mines 1979;34:70-6.

${ }^{7}$ Hankinson JL, Reger RB, Fairman RP, Lapp NL, Morgan WKC. Factors influencing expiratory flow rates in coal miners. In: Walton WH, ed. Inhaled particles IV(2). Oxford: Pergamon Press, 1977:737-52.

${ }^{8}$ Minette A. Contribution to the natural history of chronic bronchitis in coal miners. Revue de L'Institut d'Hygiène des Mines 1979;34:84-94.

${ }^{9}$ Higgins ITT. Chronic respiratory disease in mining communities. Ann NY Acad Sci 1972;200:197-210.

${ }^{10}$ Enterline PE. The effects of occupation on chronic respiratory disease. Arch Environ Health 1967;14:189-200.

${ }^{11}$ Outhred KG. Chronic bronchitis and occupation. Br Med J $1966 ; \mathrm{i}: 1232$

${ }^{12}$ Glick M, Outhred KG, McKenzie HI. Pneumoconiosis and respiratory disorders of coal mine workers of New South Wales, Australia. Ann NY Acad Sci 1972;200:316-34.

${ }^{13}$ Venizelos PC, Gerrity TR, Yeates DB. Response of human mucociliary clearance to acute alcohol administration. Arch Environ Health 1981;36:194-201.

${ }^{14}$ Lebowitz MD. Respiratory symptoms and disease related to alcohol consumption. Am Rev Respir Dis 1981;123:16-9.

${ }^{15}$ Ferris BG, Anderson DO, Zickmantel R. Prediction values for screening tests of pulmonary function. Am Rev Respir Dis 1965;91:252-61.

${ }^{16}$ Leigh J, Whitaker J. A computer based occupational health system for the coal mining industry. In: Lindberg DAB, Kaihara S, eds. Proceedings of 3rd world conference on medical informatics. Vol
2. Amsterdam: North Holland 1980:951-5.

${ }^{17}$ Cox DR. The analysis of binary data. London:Methuen, 1970:36.

${ }^{18}$ Leigh J, Outhred KG, McKenzie HI, Wiles AN. Multiple regression analysis of quantified aetiological, clinical and postmortem pathological variables related to respiratory disease in coal workers. Ann Occup Hyg 1982;26:383-400.

${ }^{19}$ Leigh J, Outhred KG, McKenzie HI, Glick M, Wiles AN. Quantified pathology of emphysema, pneumoconiosis, and chronic bronchitis in coal workers. $\mathrm{Br} J$ Ind Med 1983; 40:258-63.

${ }^{20}$ Douglas AN, Lamb D, Ruckley VA. Bronchial gland dimensions in coal miners: influence of smoking and dust exposure. Thorax 1982;37:760-4.

${ }^{21}$ Peto R, Speizer FE, Cochrane AL, et al. The relevance in adults of airflow obstruction, but not of mucus hypersecretion, to mortality from chronic lung disease. Am Rev Respir Dis 1983; 128:491-500.

${ }^{22}$ Fletcher CM, Peto R, Tinker CM, Speizer FE. The natural history of chronic bronchitis. Oxford: Oxford University Press, 1976.

${ }^{23}$ Kauffmann F, Drouet D, Lellouch J, Brille D. Twelve years spirometric changes among Paris area workers. Int $J$ Epidemiol 1979;8:201-12.

${ }^{24}$ Jacobsen M. Smoking and disability in miners. Lancet 1980;ii:740.

${ }^{25}$ Glick M, Outhred KG, McKenzie HI. The analysis of respiratory incapacity in coal mine workers of New South Wales. Med J Aust 1971;2:1136-40.

${ }^{26}$ McKenzie HI, Glick M, Outhred KG. Chronic bronchitis in coal miners: antemortem/postmortem comparisons. Thorax 1969; 24:527-35. 